University of Nebraska - Lincoln

DigitalCommons@University of Nebraska - Lincoln

8-1-2005

\title{
Thomson scattering and ponderomotive intermodulation within standing laser beat waves in plasma
}

\author{
Scott Sepke \\ University of Michigan - Ann Arbor \\ Y.Y. Lau \\ University of Michigan - Ann Arbor \\ James Paul Holloway \\ University of Michigan - Ann Arbor \\ Donald P. Umstadter \\ University of Nebraska-Lincoln, donald.umstadter@unl.edu
}

Follow this and additional works at: https://digitalcommons.unl.edu/physicsumstadter

Part of the Physics Commons

Sepke, Scott; Lau, Y.Y.; Holloway, James Paul; and Umstadter, Donald P., "Thomson scattering and ponderomotive intermodulation within standing laser beat waves in plasma" (2005). Donald Umstadter Publications. 6.

https://digitalcommons.unl.edu/physicsumstadter/6

This Article is brought to you for free and open access by the Research Papers in Physics and Astronomy at DigitalCommons@University of Nebraska - Lincoln. It has been accepted for inclusion in Donald Umstadter Publications by an authorized administrator of DigitalCommons@University of Nebraska - Lincoln. 


\title{
Thomson scattering and ponderomotive intermodulation within standing laser beat waves in plasma
}

\author{
Scott Sepke, ${ }^{*}$ Y. Y. Lau, James Paul Holloway, and Donald Umstadter ${ }^{\dagger}$ \\ Department of Nuclear Engineering and Radiological Sciences, University of Michigan, Ann Arbor, Michigan 48109-2099, USA
}

(Received 12 April 2005; published 12 August 2005)

\begin{abstract}
Electrons in a standing electromagnetic wave - an optical lattice - tend to oscillate due to the quiver and ponderomotive potentials. For sufficiently intense laser fields $\left(I \lambda^{2} \lesssim 5 \times 10^{17} \mathrm{~W} \mathrm{~cm}^{-2} \mu \mathrm{m}^{2}\right)$ and in plasmas with sufficiently low electron densities $\left(n \leqq 10^{18} \mathrm{~cm}^{-3}\right)$, these oscillations can occur faster than the plasma can respond. This paper shows that these oscillations result in Thomson scattering of light at both the laser and ponderomotive bounce frequencies and their harmonics as well as at mixtures of these frequencies. We term this mixing ponderomotive intermodulation. Here, the case of counterpropagating laser beams creating a one-dimensional (1D) optical lattice is analyzed. The near-equilibrium electron orbits and subsequent Thomson scattering patterns are computed in the single-particle limit. Scaling laws are derived to quantify the range of validity of this approach. Finally, collective plasma and laser focusing effects are included by using particlein-cell (PIC) techniques. This effect resulting in light-frequency conversion has applications both as an infrared light source and as a means to diagnose high laser intensities inside dense plasmas.
\end{abstract}

DOI: 10.1103/PhysRevE.72.026501 PACS number(s): 41.60. $-\mathrm{m}$, 42.65.Ky, 42.65.Re, 52.20.Dq

\section{INTRODUCTION}

The interaction of beating electromagnetic waves within plasma has been of interest for some time, with Rosenbluth and Liu having discussed copropagating lasers in plasma as early as 1972 [1]. Recently, the idea of overlapped laser pulses has received much attention and has been suggested for several applications. Such waves are created in many of the proposed laser accelerators such as the colliding beam accelerator [2-6] as well as in Raman amplification [7-10], optical traps [11], plasma gratings [12], and internal confinement fusion Hohlraums [13]. Laser pulses also overlap in high-density proton acceleration experiments when light is reflected from the critical surface [14].

Owing to their wide applicability, infrared light sources have also garnered much attention recently. Free electron lasers, fast diodes, thermal sources, and even tabletop laser sources have been both proposed and demonstrated as infrared generators [15-21]. Such sources have been proposed for use to image proteins, water, and even metals contained within substrate materials through direct imaging as well as pump probe experiments [22].

Generation of an ultrafast infrared light burst is a challenging problem. In this article, we demonstrate theoretically that by employing an optical lattice generated by the beat pattern of two interfering laser beams, electrons Thomson scatter the laser light into longer wavelengths. The wavelength generated is tunable with the laser intensity, $I$. For the case of counter propagating laser pulses, the emitted frequency is proportional to $I^{1 / 2}$. Moreover, this light is emitted

\footnotetext{
*Present address: Department of Physics and Astronomy, University of Nebraska, Lincoln, Nebraska 68588-0111, USA. Electronic address: ssepke2@unlserve.unl.edu

${ }^{\dagger}$ Present address: Department of Physics and Astronomy, University of Nebraska, Lincoln, Nebraska 68588-0111, USA.
}

along the laser polarization direction and is, therefore, inherently separated from the bright laser source, eliminating the problem of filtering out the laser wavelength without simultaneously removing the desired wavelength.

In this paper, we take up the topic of equal frequency and amplitude colliding laser beams and the light scattered by a test electron within these fields. This is the same laser geometry employed in the classic Kaptiza-Dirac problem, in which energetic electrons are directed across the standing light wave and subsequently diffracted $[23,24]$. In this study, however, we consider low-energy electrons trapped in the laser fields' ponderomotive potential wells. In both cases, the lasers set up a one-dimensional (1D) standing optical lattice with a high potential near the magnetic-field nodes and a low potential near the electric-field nodes. Low-energy electrons tend to bunch in the low-energy region, oscillating about the minimum with a period $\tau_{z}$. The individual electron dynamics in such a system are, of course, then dominated by both the laser period, $\tau_{0}$, and the ponderomotive bounce period, $\tau_{z}$, and as will be shown later, $\tau_{0} \lesssim \tau_{z} \ll \tau_{p}=2 \pi \omega_{p}^{-1}$ for plasma densities less than $10^{19} \mathrm{~cm}^{-3}$. This implies that these electrons respond on a time scale faster than the plasma and such single-particle effects can exist independent of the plasma. This approach illustrates a phenomenon that we have termed ponderomotive intermodulation - that is, intermodulation of the laser and ponderomotive oscillation frequencies through the optical lattice, manifested in the Thomson-scattered radiation patterns.

We begin in the first three sections by analytically calculating the electron orbits near the magnetic- and electric-field nodes, as these are equilibria. Using these results, the subsequently Thomson-scattered light is then computed as both a function of frequency and direction of observation analytically and numerically by utilizing the Larmor formula and applying Parseval's theorem, following the model used previously for both Thomson [25,26] and Compton [27-33] scattering. These results are then extended to an arbitrary 
initial electron phase, and simple scaling laws are derived to bound the range of validity of this single-particle approach. Plasma and more realistic laser fields are then added using particle-in-cell (PIC) simulation techniques. Good agreement is, indeed, found for electron densities as high as $10^{19} \mathrm{~cm}^{-3}$ and for 1- $\mu \mathrm{m}$-wavelength laser pulses as short as $300 \mathrm{fs}$ focused to $12 \mu \mathrm{m}$ as predicted by the derived scalings. This paper then concludes by illustrating the experimentally significant consequences of this phenomenon, including the tunable infrared light, anomalous spectral broadening, a laserintensity diagnostic, and tunable line emission.

\section{ELECTRON DYNAMICS}

The simplest model of a plasma neglects all collective and edge effects. Thus, to begin this study, the laser fields are modeled as monochromatic plane waves propagating in the $\pm \hat{\mathbf{Z}}$ direction such that $\mathbf{E}=\hat{\mathbf{x}} 2 a_{0} \cos (z) \cos (t)$ and $\mathbf{B}$ $=\hat{\mathbf{y}} 2 a_{0} \sin (z) \sin (t)$. Throughout this paper, the axial displacement $z$ is normalized by the laser wave number $k=\omega_{0} / c$ and time $t$ and frequency by the laser angular frequency $\omega_{0}$. Also, the laser field strength $E_{0}$ has been scaled to the normalized vector potential $a_{0}=e E_{0} / m_{0} \omega_{0} c=\left(0.85 \times 10^{-9}\right) \sqrt{I} \lambda$, where $e$ $>0$ is the unit charge, $m_{0}$ is the electron rest mass, $c$ is the speed of light, $I$ is the focused laser intensity in $\mathrm{W} \mathrm{cm}^{-2}$, and $\lambda$ is the laser wavelength in $\mu \mathrm{m}$. The equation of motion for a test electron within these fields $\mathbf{F}=q(\mathbf{E}+\vec{\beta} \times \mathbf{B})$ can then be written and rearranged to yield

$$
\begin{gathered}
\gamma \beta_{x}=-2 a_{0} \cos (z) \sin (t), \quad \gamma \beta_{y}=0, \\
\gamma \frac{d}{d t} \gamma \frac{d z}{d t}=2 a_{0}^{2} \sin (2 z) \sin ^{2}(t),
\end{gathered}
$$

where zero initial velocity has already been imposed since this is a Thomson-scattering model, $\beta_{j}$ is the velocity in the $j$ th direction normalized to $c$, and $\gamma$ is the relativistic Lorentz factor.

The far-field Thomson-scattered radiation follows directly from the electron orbits defined by these equations through the Lienard-Wiechert potentials as

$$
\frac{d^{2} \varepsilon}{d \Omega d \omega}=\varepsilon_{0}|\hat{\mathbf{n}} \times[\hat{\mathbf{n}} \times \mathbf{F}(\omega)]|^{2},
$$

where $\varepsilon$ is the radiated energy in ergs, $\varepsilon_{0}=e^{2} / 4 \pi^{2} c, \hat{\mathbf{n}}$ is the unit direction of observation, $d \Omega$ is a unit solid angle, and $d \omega$ is a unit frequency [34]. The radiated energy is normalized to $\varepsilon_{0}$ throughout $\bar{\varepsilon}=\varepsilon / \varepsilon_{0}$. The dimensionless amplitude function is, in turn, given by

$$
\mathbf{F}(\omega)=\omega \int_{-\infty}^{\infty} \vec{\beta}(t) \exp \{i \omega[t-\hat{\mathbf{n}} \cdot \mathbf{x}(t)]\} d t
$$

When $\beta$ is truly periodic with period $\tau$ and net displacement per period $\mathbf{x}_{0}$ this integral can be shown to give an infinite series of harmonics

$$
\begin{aligned}
\mathbf{F}(\omega)= & \omega \omega_{1} \int_{0}^{\tau} \vec{\beta}(t) \exp \{i \omega[t-\hat{\mathbf{n}} \cdot \mathbf{x}(t)]\} d t \\
& \times \sum_{m=-\infty}^{\infty} \delta\left(\omega-m \omega_{1}\right),
\end{aligned}
$$

where $\omega_{1}$ is the base frequency given by $2 \pi /\left(\tau-\hat{\mathbf{n}} \cdot \mathbf{x}_{0}\right)$. For $a_{0} \leqslant 0.2$, the regime studied in this paper, $\omega_{1}=\omega=2 \pi / \tau$ $[26,35]$.

To understand the conversion of laser energy to Thomsonscattered light, the orbits must be computed for all initial conditions, and these velocities Fourier-transformed in the detector-retarded time as shown earlier. Unfortunately, this is a complicated set of coupled, nonlinear equations, which are difficult to solve, in general, even numerically $[14,36]$. For low- to moderate, intensity lasers-e.g., $I \lesssim 5$ $\times 10^{17} \mathrm{~W} \mathrm{~cm}^{-2}$ for $\lambda=800 \mathrm{~nm}$ - a test electron quivers within the lattice due to the sinusoidal ponderomotive potential given by

$$
\varphi_{p}=a_{0}^{2} \cos ^{2}(z)
$$

in units of electron rest-mass energy. This implies two equilibria, one unstable at the nodes of the magnetic field $(z$ $=n \pi)$ and the other stable at the electric-field nodes $[z$ $=(2 n+1) \pi / 2]$.

\section{MAGNETIC-FIELD-NODE EQUILIBRIUM}

The nodes of the magnetic-field wave occur at $\bar{z}_{N}=N \pi$ for integer $N$. The electron motion here is purely along the laser polarization $(\hat{\mathbf{x}})$ and is known exactly for arbitrary $a_{0}$

$$
\beta_{x}(t)=\frac{-2 a_{0} \cos \left(\bar{z}_{N}\right) \sin (t)}{\sqrt{1+4 a_{0}^{2} \sin ^{2}(t)}} .
$$

This velocity corresponds to the special case of counterpropagating lasers recently calculated by Salamin et al. [33], which can be integrated directly to determine $x(t)$

$$
x(t)=\arcsin \left[\frac{2 a_{0} \cos (t)}{\sqrt{1+4 a_{0}^{2}}}\right]-\arcsin \left[\frac{2 a_{0}}{\sqrt{1+4 a_{0}^{2}}}\right] .
$$

For $a_{0}<0.5$, this can be expanded and rearranged to yield

$$
\beta_{x}(t)=-2 a_{0} \cos \left(\bar{z}_{N}\right) \sum_{\substack{p=1 \\ p \text { odd }}}^{\infty} c_{p} \sin (p t),
$$

where $c_{p}$ are known expansion coefficients, the first few of which are given in Table I. Since the oscillation period is the laser period and no net displacement occurs, the radiation pattern consists of only laser harmonics. In fact, the radiation energy per unit solid angle emitted normal to $\hat{\mathbf{x}}$ is known exactly

$$
\frac{d \bar{\varepsilon}}{d \Omega}(\hat{\mathbf{n}} \cdot \hat{\mathbf{x}}=0)=\sum_{\substack{m=1 \\ m \text { odd }}}^{\infty} 4 \pi^{2} a_{0}^{2} m^{2} c_{m}^{2},
$$

where $m$ denotes the harmonic number. Of course, since $a_{0}$ is small, only the first few harmonics are significant. The angu- 
TABLE I. The first six Fourier coefficients for describing the electron motion at the magnetic-field node in the low-field limit to order $a_{0}^{10} \cdot A_{m}=(-1)^{m} a_{0}^{2 m} \Gamma[(2 m+1) / 2] /[\sqrt{\pi} \Gamma(m+1)]$.

Expansion coefficients for the low-field radiation pattern

$$
\begin{gathered}
c_{1}=\left[1+3 A_{1}+10 A_{2}+35 A_{3}+126 A_{4}+462 A_{5}\right] \\
c_{3}=-\left[A_{1}+5 A_{2}+21 A_{3}+84 A_{4}+330 A_{5}\right] \\
c_{5}=\left[A_{2}+7 A_{3}+36 A_{4}+165 A_{5}\right] \\
c_{7}=-\left[A_{3}+9 A_{4}+55 A_{5}\right] \\
c_{9}=\left[A_{4}+11 A_{5}\right] \\
c_{11}=-\left[A_{5}\right]
\end{gathered}
$$

lar distribution of this light can also be well approximated for the first two laser harmonics as

$$
\begin{gathered}
\frac{d \bar{\varepsilon}}{d \Omega}\left(\mu, \omega_{0}\right)=4 \pi^{2} a_{0}^{2} c_{1}^{2}\left(1-\mu^{2}\right), \\
\frac{d \bar{\varepsilon}}{d \Omega}\left(\mu, 2 \omega_{0}\right)=64 \pi^{2} a_{0}^{4}\left(c_{1}+c_{3}\right)^{2} \mu^{2}\left(1-\mu^{2}\right),
\end{gathered}
$$

where $\mu=\hat{\mathbf{n}} \cdot \hat{\mathbf{x}}$. Notice the characteristic Thomson-scattering lobed structure in the angular distribution of these harmonics and the azimuthal symmetry built in by the inherent rotational invariance about $\hat{\mathbf{x}}$ of the coordinate system.

As $a_{0}$ becomes large, the velocity approximates a square wave, $\beta_{x}(t)=-\cos \left(z_{N}\right)$ for $t \epsilon(0, \pi)$ and $\cos \left(z_{N}\right)$ for $t \epsilon(\pi, 2 \pi)$. This high field orbit also results in a spectrum of laser harmonics with the higher orders becoming increasingly prominent as $a_{0}$ approaches infinity. This limiting spectrum $\left(a_{0}\right.$ $\rightarrow \infty)$ is given by

$$
\frac{d \bar{\varepsilon}}{d \Omega}=\sum_{m=-\infty}^{\infty}\left[\frac{8}{\mu_{+} \mu_{-}}-\left(\frac{\cos \left(m \mu_{-} \pi\right)}{\frac{1}{4} \mu_{-}}+\frac{\cos \left(m \mu_{+} \pi\right)}{\frac{1}{4} \mu_{+}}\right)\right]
$$

where $\mu_{ \pm}=1 \pm \hat{\mathbf{n}} \cdot \mathbf{x}$. The characteristic structure present in the low field approximation is retained and expanded to include all harmonics. Again, no emission is observed along the laser polarization and only odd harmonics are emitted normal to this, as is expected from one-dimensional, periodic motion.

\section{ELECTRIC-FIELD-NODE EQUILIBRIUM}

The magnetic-field nodes represent unstable equilibria being at local maxima of the ponderomotive potential. The nodes of the electric field, however, occur at the local minima and are, therefore, stable. The equations of motion can then be solved in a neighborhood about these points, $\bar{z}_{n}=\pi / 2+n \pi$ for integer $n$. Linearization of this system is achieved by asserting that $\gamma=1$ and expanding all sinusoids in $z$ to first order about $z_{n}$. This is equivalent to restricting the electron to nonrelativistic motion, i.e., $a_{0} \ll 1$, and approximating the minimum of the ponderomotive well quadratically. This linearization yields the Mathieu equation
TABLE II. Summary of the lowest-order solution to the linearized single-particle model near the electric-field node equilibrium. This model assumes that terms of the form $\beta_{i} \beta_{j}$ for $(i, j) \epsilon(x, y, z)$ are much less than one and that oscillations around the fixed points located at $z_{n}=(2 n+1) \pi / 2$ for $n \epsilon \mathbb{Z}$ are small. The terms $x_{0}, y_{0}$, and $\eta_{0}$ represent the electron initial conditions.

Linearized single-particle model lowest-order solution

$$
\begin{gathered}
\beta_{x}(t)=2(-1)^{n} a_{0} \sin (t) \sin \left[\eta_{0} \cos \left(\omega_{z 0} t\right)\right] \\
x(t)=(-1)^{n+1}\left(a_{0} \eta_{0} / 1-\omega_{z 0}^{2}\right)\left\{\left(1+\omega_{z 0}\right) \cos \left[\left(1-\omega_{z 0}\right) t\right]\right. \\
\left.+\left(1-\omega_{z 0}\right) \cos \left[\left(1+\omega_{z 0}\right) t\right]-2\right\}+x_{0} \\
\beta_{y}(t)=0 \\
y(t)=y_{0} \\
\beta_{z}(t)=-\sqrt{2} \eta_{0} a_{0} \sin \left(\omega_{z 0} t\right) \\
z(t)=\eta_{0} \cos \left(\omega_{z 0} t\right)+z_{n}
\end{gathered}
$$

$$
\frac{d^{2}}{d t^{2}}\left[z(t)-\bar{z}_{n}\right]+4 a_{0}^{2} \sin ^{2}(t)\left[z(t)-\bar{z}_{n}\right]=0,
$$

where $\left|z(t)-\bar{z}_{n}\right| \ll \pi / 2$. This localization is not as restrictive as it appears. PIC simulations show that electrons will, indeed, bunch near the bottom of the ponderomotive well, thereby depleting the electron population away from this linearized solution.

The orbit is found by positing a series solution in $z(t)$ such that $z(t)-\bar{z}_{n}=z_{l}(t)+a_{0}^{2} g_{2}(t)+a_{0}^{4} g_{4}(t)+\cdots$, where $z_{l}(t)$ is the low-frequency, time-averaged axial displacement, and the remaining terms are small, high-frequency corrections. The low-frequency solution is a simple harmonic motion form with characteristic ponderomotive oscillation frequency $\omega_{z 0}$ $\equiv a_{0} \sqrt{2}$, and $z_{l}(t)=\eta_{0} \cos \left(\omega_{z 0} t\right)$, where $\eta_{0} \equiv z(t=0)-\bar{z}_{n}$. The high-frequency corrections are then given by the recursion

$$
\begin{gathered}
\ddot{g}_{2}(t)=2 \eta_{0} \cos (2 t) \cos \left(\omega_{z 0} t\right) \\
\ddot{g}_{m}(t)+4 \sin ^{2}(t) g_{m-2}(t)=0,
\end{gathered}
$$

where $m=4,6,8, \ldots$. The axial velocity then follows by simple differentiation, and the transverse velocity and displacement follow by direct substitution into Eq. (1) and integration in time as shown in Table II.

The electron motion is dominated by two time scales forming so-called Lissajous patterns. The axial motion is a simple oscillation at the ponderomotive bounce frequency $\omega_{z 0}$. The transverse motion, however, is a more complicated mixture of the laser and ponderomotive bounce frequencies.

With the orbit known, this can-in principle-be Fouriertransformed according to Eqs. (3) and (4) to give the associated Thomson-scattering radiation pattern as a function of frequency $\omega$ and direction of observation $\hat{\mathbf{n}}$. The electron orbit is truly periodic and Eq. (5) can be used exactly when the field strength is chosen such that $a_{0}=\sqrt{2} /(2 N)$, where $N$ $=2,3,4, \ldots$. In that case, the base frequency $\omega_{1}$ is then simply the bounce frequency $\omega_{z 0}$. In fact, this formalism can be used for any small $a_{0}$, since the deviation from true period- 
icity is small. Numerical investigations have shown that the difference in transverse and axial periods only acts to slightly broaden the harmonics.

The emitted radiation pattern can be approximated analytically by expanding the retarded time phase term in Eq. (4) as

$$
e^{-i \hat{\mathbf{n}} \cdot \mathbf{x}} \approx 1-i \hat{\mathbf{n}} \cdot \mathbf{x} .
$$

Performing the integration of Eq. (3) in this way yields scattered radiation at both the bounce and laser fundamental frequencies as well as their second harmonics

$$
\begin{gathered}
\frac{d \bar{\varepsilon}}{d \Omega}\left(\omega_{z}\right)=\pi^{2} \eta_{0}^{2} \omega_{z}^{2}\left(1-n_{z}^{2}\right) \\
\frac{d \bar{\varepsilon}}{d \Omega}\left(2 \omega_{z}\right)=2 \pi^{2} a_{0}^{2} \eta_{0}^{4}\left(2 \omega_{z}\right)^{2}\left[n_{z}^{2}\left(1-n_{z}^{2}\right)-2 n_{x}^{2} n_{z}^{2}\left(\frac{\omega_{z}^{2}}{1-\omega_{z}^{2}}\right)\right. \\
\left.+n_{x}^{2}\left(1-n_{x}^{2}\right)\left(\frac{\omega_{z}^{2}}{1-\omega_{z}^{2}}\right)^{2}\right] \\
\frac{d \bar{\varepsilon}}{d \Omega}\left(\omega_{0}\right)=\frac{1}{2} \pi^{2} \eta_{0}^{4}\left[n_{z}^{2}\left(1-n_{x}^{2}\right)-2 n_{x}^{2} n_{y}^{2} n_{z}^{2}\left(\frac{\omega_{z}^{2}}{1-\omega_{z}^{2}}\right)+n_{x}^{2}\left(1-n_{z}^{2}\right)\right. \\
\left.\times\left(\frac{\omega_{z}^{2}}{1-\omega_{z}^{2}}\right)^{2}\right] \\
\frac{d \bar{\varepsilon}}{d \Omega}\left(2 \omega_{0}\right)=4 \pi^{2} \eta_{0}^{4}\left(\frac{\omega_{z}}{1-\omega_{z}^{2}}\right)^{2} n_{x}^{2}\left(1-n_{x}^{2}\right)
\end{gathered}
$$

The second harmonic of the ponderomotive frequency scales as $a_{0}^{2} \eta_{0}^{4} \lll 1$. Thus, this emission line is not observed in numerical simulations of this problem.

The laser and ponderomotive bounce frequencies are transferred directly to the spectrum through the fast electron quiver motion and the slower ponderomotive well oscillation, thereby generating the aforementioned harmonics. These frequencies also couple through a process we have termed ponderomotive intermodulation, creating the sideband products $\omega=\omega_{0} \pm \omega_{z 0}$ and $\omega=\omega_{0} \pm 2 \omega_{z 0}$, as well as $\omega$ $=2 \omega_{0} \pm 2 \omega_{z 0}$, as given by

$$
\begin{gathered}
\frac{d \bar{\varepsilon}}{d \Omega}\left(\omega_{0} \pm \omega_{z}\right)=\frac{1}{2} \pi^{2} \eta_{0}^{2}\left(1 \pm \omega_{z}\right)^{2}\left(1-n_{x}^{2}\right), \\
\frac{d \bar{\varepsilon}}{d \Omega}\left(\omega_{0} \pm 2 \omega_{z}\right)= \\
\frac{1}{8} \pi^{2} \eta_{0}^{4}\left(1 \pm 2 \omega_{z}\right)^{4}\left[n_{z}^{2}\left(1-n_{x}^{2}\right)\right. \\
\left.\mp 2 n_{x}^{2} n_{z}^{2}\left(\frac{\omega_{z}}{1 \pm \omega_{z}}\right)+n_{x}^{2}\left(1-n_{z}^{2}\right)\left(\frac{\omega_{z}}{1 \pm \omega_{z}}\right)^{2}\right],
\end{gathered}
$$

$$
\frac{d \bar{\varepsilon}}{d \Omega}\left(2 \omega_{0} \pm 2 \omega_{z}\right)=\frac{1}{2} \pi^{2} a_{0}^{2} \eta_{0}^{4}\left(2 \pm 2 \omega_{z}\right)^{2} n_{x}^{2}\left(1-n_{x}^{2}\right) .
$$

This intermodulation mechanism is, in fact, analogous to many classical nonlinear optical phenomena, including Raman scattering. The test electron couples to both the incident laser field and a second periodic potential. In ponderomotive intermodulation, this potential is the ponderomotive potential $\varphi_{p}$, instead of the plasma-wave electrostatic potential responsible for Raman scattering [37]. Thus, intermodulation will exist whenever quiver motion driven by a laser interacts with an additional periodic potential, and ponderomotive intermodulation occurs when that potential is due to the interference of overlapping laser pulses.

Inclusion of the high-frequency corrections of Eqs. (15) and (16) acts to add additional harmonics of these two frequencies. For example, the first correction as defined by Eq. (15) yields emission up to the sixth harmonic of the laser frequency as well as the first two intermodulation products, $N \omega_{0} \pm \omega_{z 0}$ and $N \omega_{0} \pm 2 \omega_{z 0}$. These higher-order modes, however, scale strongly with the small parameter $a_{0}$ and are not observed in numerical studies.

These formulas for the Thomson spectra correctly predict the scattered frequencies and the qualitative behavior of the angular distributions of each emitted line. The approximation of Eq. (17), however, fails to capture enough detail to accurately determine the absolute magnitude of the scattered signal. By integrating Eq. (3), including the full retarded time phase term $\exp (-i \hat{\mathbf{n}} \cdot \mathbf{x})$, these magnitudes are obtained to good approximation, as discussed later.

\section{VALIDITY OF THE SINGLE-PARTICLE MODEL}

The electron dynamic equations and the resulting spectra have relied on several seemingly stringent assumptionszero plasma density and no gradients in the laser envelope. The question of the range of validity of this model then naturally arises. In making the near-equilibrium linearization at the electric-field nodes, the ponderomotive potential was modeled quadratically in $z$. Electrons initially residing farther from the node sample a greater portion of the ponderomotive well and, hence, their bounce frequencies acquire an initial phase, $\eta_{0}$, dependence. This can be calculated in the nonrelativistic limit by averaging Eq. (2) over a laser cycle and solving the resulting pendulum equation to yield

$$
\tau_{z}=2 \pi / \omega_{z}=\tau_{z 0} F\left(\cos \left(\eta_{0}\right), \pi / 2\right),
$$

where $\tau_{z}$ is the bounce period, $\omega_{z}$ is the bounce angular frequency, $\tau_{z 0}=2 \pi \omega_{z 0}^{-1}$ is the linearized bounce period, $\eta_{0}$ retains the meaning cited earlier, and $F(k, \pi / 2)$ is the elliptic integral of the first kind [38].

PIC simulations using the program OOPICPRO show that for initially cold plasmas with electron densities as high as $10^{19} \mathrm{~cm}^{-3}$, electrons will tend to bunch to densities up to an order of magnitude greater than the background plasma density in a range of one-eighth the laser wavelength about the electric-field nodes $[39,40]$. In the notation of the solutions given earlier, this means that the majority of electrons have initial phases such that $\left|\eta_{0}\right|$ ranges from 0 to $\pi / 8$. Approximating Eq. (25) using this restriction yields

$$
\tau_{z}=\tau_{z 0}\left\{1+\sum_{n=1}^{\infty}\left[\frac{1 \times 3 \times \cdots(2 n-1)}{2 \times 4 \times \cdots(2 n)}\right]^{2} \cos ^{2 n}\left(\eta_{0}\right)\right\} .
$$

This then gives a maximum increase of ponderomotive frequency of $18.03 \%$ due to initial phase variation. This also 


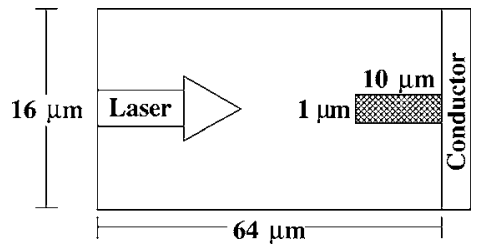

FIG. 1. Schematic diagram of the OOPICPRO simulation box. The simulation was performed in two-dimensional rectangular coordinates.

provides an estimate for the upper bound of plasma densities for which this single-particle theory is valid. Single-particle effects remain independent of collective plasma behavior provided they operate on faster time scales than the response time of the bulk plasma. Since the plasma time scale is given by $\tau_{p}=2 \pi \omega_{p}^{-1}$ and ponderomotive intermodulation operates over a time $\tau_{z} \gtrsim \tau_{0}$, this single-particle approach applies when $\tau_{z} \ll \tau_{p}$. Taking the maximum value of $\tau_{z}=1.1803 \tau_{z 0}$

$$
n_{0}\left(\mathrm{~cm}^{-3}\right) \ll\left(1.6 \times 10^{21}\right)\left(\frac{a_{0}}{\lambda(\mu \mathrm{m})}\right)^{2},
$$

where $\lambda$ is the laser wavelength given in $\mu \mathrm{m}$. For example, for a Nd:glass laser operating at $\lambda=1.053 \mu \mathrm{m}$ and a strength of $a_{0}=0.2$, the density must be less than about 5 $\times 10^{18} \mathrm{~cm}^{-3}$.

The plane-wave model of the laser also gives rise to physical limits of applicability. For the temporal profile of the laser pulse to not significantly affect this phenomenon, the radiating electron must be able to traverse several bounce periods without the field strength changing significantly. Thus, the pulse duration $\tau_{l}$ must satisfy the relation $\tau_{l} \gg \tau_{z}$. This leads to the more specific requirement

$$
\tau_{l}(\mathrm{fs}) \gg\left[\frac{5(1.1803) \sqrt{2}}{3}\right]\left[\frac{\lambda(\mu \mathrm{m})}{a_{0}}\right] .
$$

Again, for a $1.053-\mu \mathrm{m}$ laser operating at $a_{0}=0.2$, the pulse duration must be greater than approximately $100 \mathrm{fs}$. Similarly, during a bounce period, an electron can travel no more than a distance $c \tau_{z}$. For the laser field to be nearly constant to an electron traveling this distance transverse to the laser, the waist $w_{0}$ must satisfy

$$
\frac{w_{0}}{\lambda} \gg \frac{1.1803}{a_{0} \sqrt{2}},
$$

which implies a $\gtrsim 10$ - $\mu \mathrm{m}$ waist for the sample laser parameters already considered.

\section{PARTICLE-IN-CELL SIMULATIONS}

Since only the electron dynamics need be known, this problem lends itself well to particle-in-cell modeling. Simulations have been run using OOPICPRO to span the parameter space as defined in Eqs. (27)-(29), looking specifically for the effects of the bulk plasma, a finite initial temperature, finite duration, and focused laser pulses [39,40]. All of the runs consist of a rectangular simulation box $64 \mu \mathrm{m}$ in the

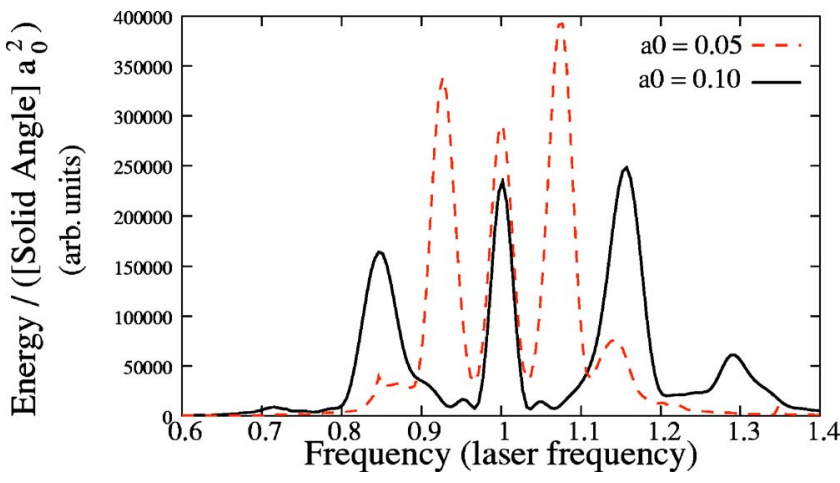

FIG. 2. (Color online) Forward (z) light as a function of the laser strength, $a_{0}$. The dashed line corresponds to $a_{0}=0.05$, and the solid line to $a_{0}=0.10$. The laser is $300 \mathrm{fs}$ in duration and focused to a waist of $14 \mu \mathrm{m}$. The background plasma density is $10^{18} \mathrm{~cm}^{-3}$. A total of 116 test-particle spectra have been summed.

laser propagation direction and $16 \mu \mathrm{m}$ transverse to this, as shown in Fig. 1. A single 300-fs laser pulse of wavelength $1 \mu \mathrm{m}$ and focused to $w_{0}=14 \mu \mathrm{m}$ is launched from the $z=0$ wall (left boundary) and propagates to the far wall, which is a perfect conductor. Here the pulse is reflected, setting up a counterpropagating geometry. Cold electrons are loaded throughout the entire simulation space, and an immobile neutralizing ion background is included. A set of 116 test electrons are randomly selected in a $10-\mu \mathrm{m}-\mathrm{by}-1-\mu \mathrm{m}$ area within the foci of the pulses.

The initial laser pulse is allowed to propagate across the $64 \mu \mathrm{m}\left(213 \frac{1}{3} \mathrm{fs}\right)$ and reflect back an additional $30 \mu \mathrm{m}$ (100 fs). OOPICPRO was modified so that the positions and velocities of a random set of test electrons are tabulated in a single output file 24 times per laser cycle for $100 \mathrm{fs}$ while the pulses are overlapped. These data are then numerically integrated according to Eq. (3) to yield the light scattered by each electron. Each signal is then incoherently summed with the other particles, producing an integrated signal. Two such spectra are shown in Fig. 2. Both of these calculations show the light scattered in the $+\hat{\mathbf{z}}$ direction. Notice that the spacing between the laser fundamental and the intermodulation products increases linearly with $a_{0}$, as predicted.

Figure 3 shows the light scattered from 116 test particles in the $x-z$ plane for $a_{0}=0.2$. The left-hand figure shows the light radiated $45^{\circ}$ above the $z$ axis, and the right-hand plot is the light scattered along the laser polarization. Notice the strong low-frequency signal along the polarization vector. For the case of a $1-\mu \mathrm{m}$ laser, this corresponds to $3.54-\mu \mathrm{m}$ light with an intensity comparable to that of the fundamental scattered in the laser propagation direction. The top scatter image also shows weak emission at roughly the frequencies $2 \omega_{0} \pm \omega_{z}$, which is predicted only when the first highfrequency correction is included in the particle orbits [see Eq. (15)]. The small laser fundamental signal along the polarization is due to the spread in laser-wave vectors (k), which is due to the laser focusing.

The analytic approximations for the Thomson-scattered light derived in Eqs. (18)-(24) correctly predict the frequency and overall angular distribution illustrated in Figs. 2 and 3-that is, a strong laser fundamental as well as the 

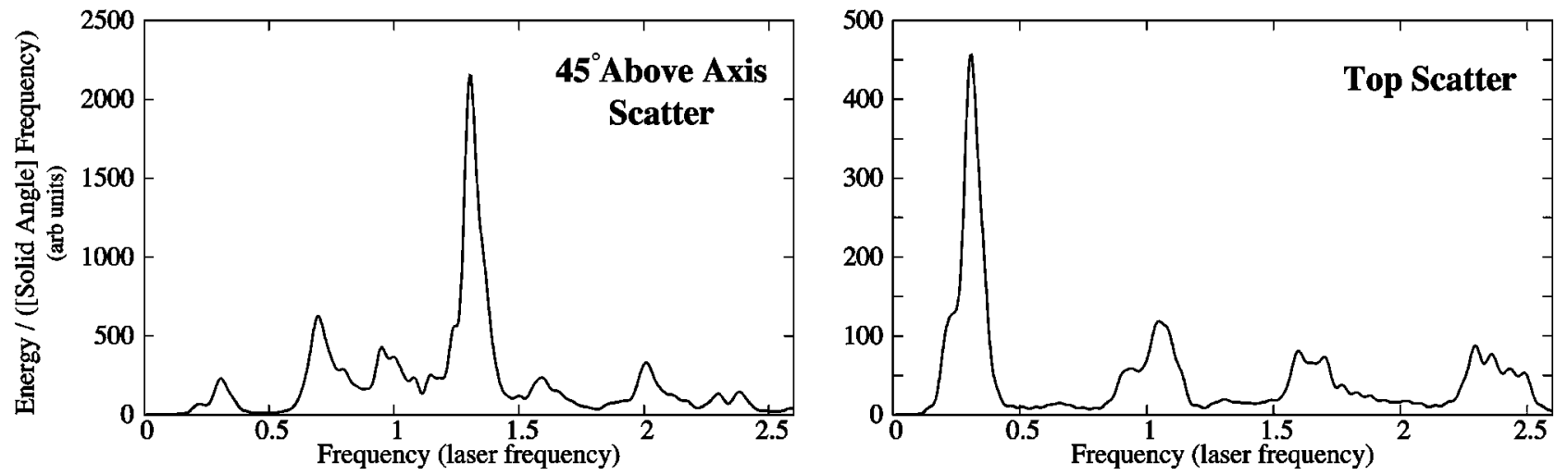

FIG. 3. Thomson scattered light in the $x$ - $z$ plane. The same plasma and laser conditions as in Fig. 2 have been used with $a_{0}=0.2$, and 116 test particles have been summed. The left-hand figure shows the spectrum along $\mathbf{n}=(\sqrt{2} / 2)(\mathbf{x}+\mathbf{z})$. The peaks correspond to the frequencies $\omega_{z}, \omega_{0}-\omega_{z}, \omega_{0}, \omega_{0}+\omega_{z}, \omega_{0}+2 \omega_{z}$ (weak), and $2 \omega_{0}$. The right-hand plot is taken along the laser polarization $(\mathbf{n}=\mathbf{x})$. The frequencies are $\omega_{z}$, $\omega_{0}, 2 \omega_{0}-\omega_{z}$, and $2 \omega_{0}+\omega_{z}$.

intermodulation products $\omega_{0} \pm \omega_{z}$ and $\omega_{0} \pm 2 \omega_{z}$ along the $\mathbf{k}$ vector, and a dominant $\omega_{z}$ signal along the laser polarization direction. The relative magnitudes of these lines predicted by this model, however, are poorly matched to the full simulation results. Figure 4 compares the forward-scattered light for 57 particles under the same laser conditions as Fig. 2 and $a_{0}=0.05$ using the PIC data (solid line) and the singleelectron orbits, now including the entire retarded-time phase term $\exp (-i \hat{\mathbf{n}} \cdot \mathbf{x})$, using 57 evenly spaced initial phases $\eta_{0}$ between $\pi / 4$ and $3 \pi / 4$ (dashed line). The agreement is quite good except that the PIC simulation produces a much larger laser fundamental signal. This can be attributed to the contribution from electrons in regions of marginal pulse overlap. These particles see only a single laser and, therefore, radiate only at this frequency [28].

Since the laser pulse propagates through the plasma before reflecting and creating the standing laser wave, a plasma wave is created, and the test electrons now no longer have a zero initial velocity when entering the standing wave. Examining the spectra radiated by PIC electrons and electrons whose equation of motion has been solved numerically given

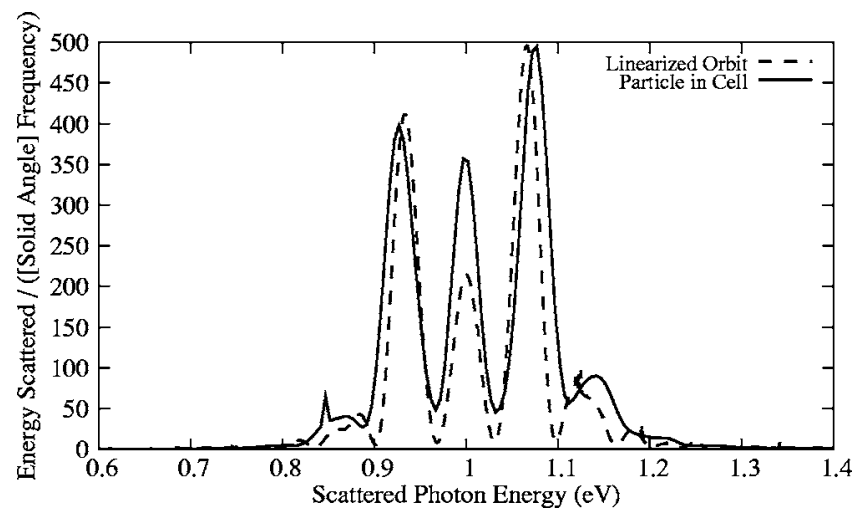

FIG. 4. A comparison of the spectra from 57 particles using the PIC simulation (solid line) and the single-particle equation of motion (dashed line). The relative agreement for each of the intermodulation products is good. The laser fundamental line is enhanced in the PIC simulation due to particles in regions of poor overlap. a small initial velocity shows that the intermodulation lines tend to be slightly offset from their theoretically predicted value due to this initial motion. Taking the ensemble as shown in these PIC results, the emitted lines tend to be slightly broadened due to the finite plasma temperature.

\section{EXPERIMENTAL SIGNATURES AND CONSEQUENCES}

By employing single-particle as well as particle-in-cell numerical techniques, the process of ponderomotive intermodulation and its potential as a light source have been investigated theoretically. Ponderomotive intermodulation has several signature characteristics amenable to laboratory measurement. The intermodulation frequencies that straddle the laser harmonics are intensity-dependent. That is, since the laser intensity $I$ scales like $I \sim a_{0}^{2}$, the bounce frequency then goes as $\omega_{z} \sim I^{1 / 2}$, and the frequency separation between harmonics and intermodulations then also scales as $I^{1 / 2}$. Thus, by simply varying the laser intensity, the intermodulation products produced by this process will shift in frequency. Conversely, this process could be used to deduce the laser intensity by measuring the absolute offset of the intermodulation products. This would be important, for example, in a dynamic Hohlraum.

In addition to intermodulation products, the preceding analysis and simulations show that the one-dimensional optical lattice created by overlapping two counterpropagating laser beams generates a significant long-wavelength signal along the laser polarization, as illustrated by Eq. (18). This light, for example, occurs at $3.76 \mu \mathrm{m}$ for a $\mathrm{Nd}$ :Glass laser operating at $1.053 \mu \mathrm{m}$ and $a_{0}=0.2$. As this top-scattered frequency scales with $a_{0}\left(\sim I^{1 / 2}\right)$, the absolute frequency is tunable by simply adjusting the laser intensity. Emission at this frequency will only occur as long as the lattice exists, that is, as long as the laser pulses are overlapped. The duration of this light source, then, is determined by the laser pulse duration-which, using chirped pulse amplification, can now easily be on the order of hundreds of femtoseconds. Thus, the optical lattice created by two overlapped counterpropa- 


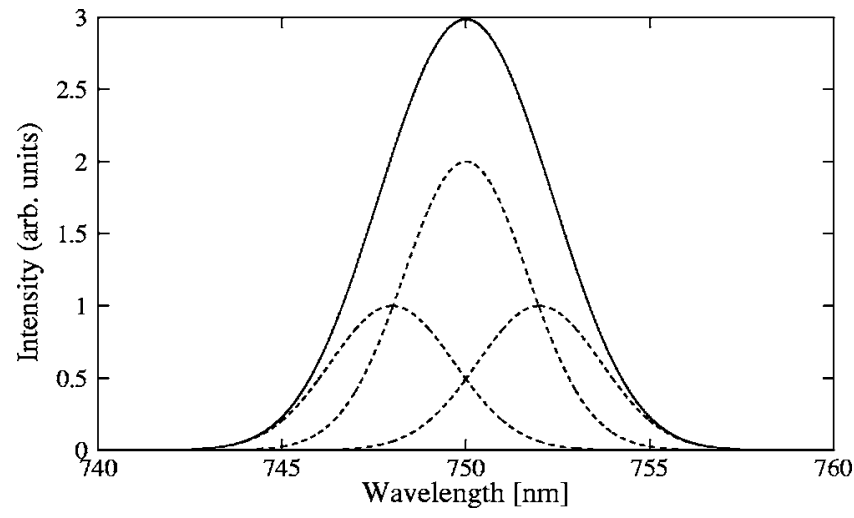

FIG. 5. The sum of three offset Gaussian signals yields a broader bell-shaped signal, which-if ponderomotive intermodulation is not accounted for-can lead to incorrect pulse-duration estimates. The three Gaussians (dashed curves) all have FWHM of $4.00 \mathrm{~nm}$. The sum of these three (solid curve), however, has a FWHM of $5.37 \mathrm{~nm}$.

gating laser pulses acts as an ultrafast, all-laser-driven, nearinfrared source. Using the PIC results shown in Fig. 3, the instantaneous scattered power radiated into a cone with slant angle $\varphi_{m}$ by a laser with a duration of $\tau$ fs, an energy of $E$ Joules incident on a plasma with an electron number density of $n_{e}$ in $\mathrm{cm}^{-3}$ is given by

$$
\begin{aligned}
P(\mathrm{~W})= & \left(10^{-20}\right)[\tau(\mathrm{fs})][E(\mathrm{~J})]\left[n_{e}\left(\mathrm{~cm}^{-3}\right)\right]\left(\frac{8 \varphi_{m}}{3}\right) \\
& \times\left.\sin \varphi_{m}\left(3-\sin ^{2} \varphi_{m}\right) \int_{\omega_{z}-\Delta \omega}^{\omega_{z}+\Delta \omega} \frac{d^{2} \bar{\varepsilon}}{d \Omega d \omega}\right|_{P I C} d \omega,
\end{aligned}
$$

where $\Delta \omega \approx 0.15$ is half of the bandwidth of the $\omega_{z}$ line normalized to the laser frequency, and the spectral energy density inside the integral is that shown in Fig. 3. Using two 1 -ps laser pulses containing $20 \mathrm{~J}$ of energy each, incident on a cold plasma with $n_{e}=3 \times 10^{18} \mathrm{~cm}^{-3}$ and assuming a detector acceptance angle of $5^{\circ}$ yields a power of $\sim 616 \mathrm{~W}$ at $3.76 \mu \mathrm{m}$. This power is low, but the tunability as well as the ultrashort duration and directionality of such an opticallattice source is encouraging. By utilizing other angles of incidence, higher intensities and, therefore, higher scattered powers may be reached.

The range of initial phases as well as the finite initial temperature of any real set of electrons tend to broaden all of the Thomson-scattered lines, as illustrated in the PIC simulation results shown in Figs. 2 and 3. When all of these factors are properly tuned, the first intermodulation products $\omega_{0} \pm \omega_{z}$ can overlap the laser fundamental mode, $\omega_{0}$, anomalously broadening the $\omega_{0}$ signal, as illustrated in Fig. 5. This can lead to incorrect pulse-duration calculations. For example, an experiment utilizing counterpropagating, 750-nm laser pulses with $a_{0} \sim 0.002$ generates a bounce wavelength span from 2.15 to $2.50 \mathrm{~nm}$, with the range being due to the spread in initial phases $\eta_{0}$. Including both the $\eta_{0}$ spread and a 4-nm full width at half maximum (FWHM) bandwidth of the laser-which is also imparted to the sidebands - the laser and first intermodulation product signals overlap, which pre- cludes pulse lengthening from being observed using bandwidth techniques. Such broadened pulses may also lend themselves to chirped pulse amplification as well and need to be taken into consideration in the interpretation of data ascribed to other broadening mechanisms, such as relativistic self-modulation.

Additionally, such spectrally broadened pulses hold the potential to be temporally compressed. This process is analogous to many parametric amplification processes, but now the laser fields themselves are being used as the nonlinear medium. For such a process to work, however, the phase characteristics of the Thomson-scattered light also must be considered.

Ponderomotive intermodulation also generates a strong $\omega_{0}+\omega_{z}$ signal in the near-forward/near-backward direction, which can mimic a broad $\sim 3 / 2$ harmonic of a $1-\mu \mathrm{m}$ laser. The broadness of such emission is, of course, caused both by the temporal and spatial variation of a Gaussian laser pulse and by the finite range of $\eta_{0}$ values in a plasma. This $\sim 3 / 2$ harmonic light can be further enhanced by the weaker $2 \omega_{0}$ $-2 \omega_{z}$ scattering when $a_{0} \sim 0.2$. Similar features have been observed experimentally [41].

\section{SUMMARY AND CONCLUSIONS}

We have here derived the equilibrium electron orbits in a one-dimensional optical-lattice setup by counterpropagating laser pulses of equal amplitude and frequency. This motion, in general, includes both fast laser quiver motion and slower ponderomotive oscillations. These time scales mix to yield harmonics of both the laser and ponderomotive frequencies as well as several intermodulation products. Emission of the ponderomotive bounce frequency has been shown to have significant potential as an ultrafast near-infrared source.

For completeness, the spectra emitted at the magneticfield nodes have also been computed. The slow, axial bounce motion is seen to disappear, and the orbit and, hence, the radiation pattern then no longer show mixing but only emission of laser harmonics. As this is an unstable equilibrium point, however, the ponderomotive bounce motion will dominate the radiated light.

Ponderomotive intermodulation occurs independent of the plasma response and is, therefore, significant in realistic laboratory conditions, as demonstrated in Eqs. (27)-(29). The resulting scattered light produces-in addition to the low-frequency emission peaked along the laser polarization-intensity-dependent spectral satellites about the laser harmonics in much the same way Raman scattering does through the plasma wave. This not only gives a signature dependence for diagnosis of this effect and the laser intensity in the plasma but can also lead to anomalous spectral broadening of laser harmonics.

In many plasma problems, multiple time scales act to drive electron motion. Associated with each mode is a natural frequency, which interacts with the others to produce the resulting orbit. This mixing can be seen directly through the Thomson-scattered light since the Larmor formula as shown 
in Eqs. (3) and (4) is nothing more than a Fourier decomposition of the electron velocity. We have here demonstrated a particular case of such intermodulation driven by the ponderomotive potential of beating laser beams. The idea can now be extended to specific geometries and even other driving mechanisms to extend this method to additional scattered wavelengths.

\section{ACKNOWLEDGMENTS}

The authors would like thank Kirk Flippo, Sudeep Banerjee, and Matthew Rever for many stimulating conversations. This work has been supported by Sandia National Laboratories, the U.S. Department of Energy, the National Science Foundation, and the Office of Naval Research.
[1] M. N. Rosenbluth and C. S. Liu, Phys. Rev. Lett. 29, 701 (1972).

[2] G. Shvets, N. J. Fisch, A. Pukhov, and J. Meyer-ter-Vehn, Phys. Rev. E 60, 2218 (1999).

[3] G. Shvets and N. J. Fisch, Phys. Rev. Lett. 86, 3328 (2001).

[4] G. Shvets, N. J. Fisch, and A. Pukhov, Phys. Plasmas 9, 2383 (2002).

[5] C. S. Liu and V. K. Tripathi, Phys. Plasmas 9, 3995 (2002).

[6] K. Nagashima, J. Koga, and M. Kando, Phys. Rev. E 64, 066403 (2001).

[7] G. Shvets, N. J. Fisch, A. Pukhov, and J. Meyer-ter-Vehn, Phys. Rev. Lett. 81, 4879 (1998).

[8] V. M. Malkin, G. Shvets, and N. J. Fisch, Phys. Rev. Lett. 82, 4448 (1999).

[9] V. M. Malkin, G. Shvets, and N. J. Fisch, Phys. Rev. Lett. 84, 1208 (2000).

[10] Y. Ping, I. Geltner, N. J. Fisch, G. Shvets, and S. Suckewer, Phys. Rev. E 62, R4532 (2000).

[11] P. Zhang, N. Saleh, S. Chen, Z. M. Sheng, and D. Umstadter, Phys. Rev. Lett. 91, 225001 (2003).

[12] J. C. Fernandez, J. A. Cobble, B. H. Failor, D. F. DuBois, D. S. Montgomery, H. A. Rose, H. X. Vu, B. H. Wilde, M. D. Wilke, and R. E. Chrien, Phys. Rev. Lett. 77, 2702 (1996).

[13] Z. M. Sheng, J. Zhang, and D. Umstadter, Appl. Phys. B 77, 673 (2003)

[14] Y. Sentoku, V. Bychenkov, K. Flippo, A. Maksimchuk, K. Mima, G. Mourou, Z. Sheng, and D. Umstadter, Appl. Phys. B 74, 207 (2002).

[15] G. L. Carr, M. C. Martin, W. R. McKinney, K. Jordan, G. R. Neil, and G. P. Williams, Nature 420, 153 (2002).

[16] G. Ramian, Nucl. Instrum. Methods Phys. Res. A 318, 225 (1992).

[17] D. W. Porterfield, T. W. Crowe, R. F. Bradley, and N. R. Erickson, IEEE Trans. Microwave Theory Tech. MTT-47, 419 (1999).

[18] P. H. Siegel, IEEE Trans. Microwave Theory Tech. MTT-50, 910 (2002)

[19] D. H. Auston, K. P. Cheung, J. A. Valdmanis, and D. A. Kleinman, Phys. Rev. Lett. 53, 1555 (1984).

[20] A. Bonvalet, M. Joffe, J. L. Martin, and A. Migus, Appl. Phys. Lett. 67, 2907 (1995).

[21] D. You, R. R. Jones, P. H. Bucksbaum, and D. R. Dykaar, Opt. Lett. 18, 290 (1993).
[22] Q. Chen and X. C. Zhang, in Ultrafast Laser Technology and Applications, edited by M. E. Fermann, A. Galvanauskas, and G. Sucha (Marcel Dekker, New York, 2001), pp. 521-572.

[23] P. L. Kapitza and P. A. M. Dirac, Proc. Cambridge Philos. Soc. 29, 297 (1933).

[24] D. L. Freimund, K. Aflatooni, and H. Batelaan, Nature 413, 132 (2001).

[25] E. S. Sarachik and G. T. Schappert, Phys. Rev. D 1, 2738 (1970).

[26] F. He, Y. Lau, D. Umstadter, and T. Strickler, Phys. Plasmas 9, 4325 (2002).

[27] Y. I. Salamin and F. H. M. Faisal, Phys. Rev. A 54, 4383 (1996).

[28] F. He, Y. Y. Lau, D. P. Umstadter, and R. Kowalczyk, Phys. Rev. Lett. 90, 055002 (2003).

[29] F. V. Hartemann, Phys. Plasmas 5, 2037 (1998).

[30] F. V. Hartemann, A. L. Troha, N. C. Luhmann, and Z. Toffano, Phys. Rev. E 54, 2956 (1996).

[31] Y. I. Salamin and F. H. M. Faisal, Phys. Rev. A 61, 043801 (2000).

[32] Y. I. Salamin and F. H. M. Faisal, Phys. Rev. A 55, 3964 (1997).

[33] Y. I. Salamin, G. R. Mocken, and C. H. Keitel, Phys. Rev. E 67, 016501 (2003).

[34] J. D. Jackson, Classical Electrodynamics, 6th ed. (John Wiley, New York, 1962), 661-697.

[35] Y. Lau, F. He, D. Umstadter, and R. Kowalczyk, Phys. Plasmas 10, 2155 (2003).

[36] Z.-M. Sheng, K. Mima, Y. Sentoku, M. S. Jovanovic, T. Taguchi, J. Zhang, and J. Meyer-ter-Vehn, Phys. Rev. Lett. 88, 055004 (2002).

[37] W. Kruer, The Physics of Laser Plasma Interactions Frontiers in Physics (Addison-Wesley Publishing, Reading, MA, 1988), pp. 60-62.

[38] C. H. Edwards and D. E. Penny, Differential Equations (Prentice-Hall, Englewood Cliffs, NJ, 1996).

[39] D. L. Bruhwiler, R. E. Giacone, J. R. Cary, J. P. Verboncoeur, P. Mardahl, E. Esarey, W. P. Leemans, and B. A. Shadwick, Phys. Rev. ST Accel. Beams 4, 101302 (2001).

[40] D. L. Bruhwiler, D. A. Dimitrov, J. R. Cary, E. Esarey, W. Leemans, and R. E. Giacone, Phys. Plasmas 10, 2022 (2003).

[41] S. Banerjee (private communication). 